\title{
The prognostic role of a combined fibrinogen and inflammation- based index in patients with metastatic breast cancer
}

\author{
Qiuge Liu ${ }^{1,2 \#}$, Shuangshuang Fang ${ }^{1,3 \#}$, Shanshan Liang ${ }^{1,2 \#}$, Jinyan Lv $^{1,2}$, Gang Wang ${ }^{1,2}$, Rongbin Tang ${ }^{1,2}$, \\ Xuening $\mathrm{Ji}^{1,2}$, Tong Zhao ${ }^{1,2}$, Jiaoyang $\mathrm{Li}^{1,2}$, Lu Xu ${ }^{1,2}$, Lianli Ma ${ }^{1,2}$, Ruoyu Wang ${ }^{1,2}$, Heming Li ${ }^{1,2}$ \\ ${ }^{1}$ Department of Oncology, Affiliated Zhongshan Hospital of Dalian University, Dalian, China; ${ }^{2}$ The Key Laboratory of Biomarker High Throughput \\ Screening and Target Translation of Breast and Gastrointestinal Tumor, Dalian, China; ${ }^{3}$ Department of Oncology, the Third Affiliated Hospital of \\ Zunyi Medical University, Zunyi, China \\ Contributions: (I) Conception and design: H Li, Q Liu, S Liang, R Wang; (II) Administrative support: J Li; (III) Provision of study materials or \\ patients: J Li, G Wang, R Tang; (IV) Collection and assembly of data: T Zhao, X Ji; (V) Data analysis and interpretation: J Li, L Xu, L Ma; (VI) \\ Manuscript writing: All authors; (VII) Final approval of manuscript: All authors. \\ \#These authors contributed equally to this work. \\ Correspondence to: Heming Li; Ruoyu Wang. Department of Medical Oncology, Affiliated Zhongshan Hospital of Dalian University, No. 6 Jiefang \\ Street, Dalian, 110006, China. Email: liheming8563@hotmail.com; wangruoyu1963@hotmail.com.
}

Background: The activation of inflammation and coagulation cascades plays an essential role in the development of various malignancies, including metastatic breast cancer (MBC). This retrospective study aimed to investigate the prognostic role of the combination of fibrinogen and the inflammation-based index in patients with MBC.

Methods: A total of 176 patients with MBC were retrospectively reviewed. The clinical and pathological data of included patients were followed-up and analyzed. The plasma fibrinogen concentration (FIB), neutrophil-lymphocyte ratio (NLR), and platelet-lymphocyte ratio (PLR) were measured. Dynamic variations in the FIB, NLR, and PLR values were collected from $56 \mathrm{MBC}$ patients before and after firstline therapy. Receiver operating characteristic (ROC) curves were constructed to assess the optimal cut-off values. Correlations between FIB and NLR or PLR were evaluated using Spearman correlation analysis. The Kaplan-Meier method, two-tailed log-rank test, and Cox proportional hazard model were used for statistical analysis.

Results: Baseline FIB was positively correlated with NLR and PLR values in MBC patients $(\mathrm{P}<0.05)$. Additionally, multivariable analysis proved that the ERBB2 + subtype $(\mathrm{P}=0.023)$, basal-like subtype $(\mathrm{P}=0.032)$, targeted therapy $(\mathrm{P}=0.033)$, other regimens $(\mathrm{P}=0.005)$, and baseline FIB level $(\mathrm{P}=0.004)$ were independent prognostic variables for progression-free survival (PFS) in MBC patients. Furthermore, ERBB2+, basal-like subtypes, and baseline hyperfibrinogenemia were independent factors for poor prognosis in MBC patients [hazard ratio (HR): 3.717, 95\% confidence interval (CI): 1.561-8.851, P=0.003; HR: 3.245, 95\% CI: $1.368-$ 7.698, P=0.008; HR: 2.069, 95\% CI: 1.352-3.167, P=0.001, respectively]. Most importantly, the FIB level increased significantly after first-line therapy in patients with disease progression $(3.73 \pm 0.63 v s .5 .32 \pm 0.52 \mathrm{~g} / \mathrm{L}$, $\mathrm{P}=0.042)$ and also decreased markedly in stable disease $(3.42 \pm 1.05$ vs. $3.03 \pm 0.73 \mathrm{~g} / \mathrm{L}, \mathrm{P}=0.036)$. However, PFS and overall survival (OS) were not significantly correlated with the dynamic changes of FIB and the inflammation-based index.

Conclusions: The present study provided evidence that baseline FIB combined with NLR and PLR could serve as prognostic predictors for MBC patients. Dynamic change of FIB before and after first-line therapy could also be used as a potential predictor of therapeutic response in MBC patients.

Keywords: Metastatic breast cancer (MBC); fibrinogen (FIB); neutrophil lymphocyte ratio (NLR); platelet lymphocyte ratio (PLR); prognosis 
Submitted May 22, 2020. Accepted for publication Sep 17, 2020.

doi: $10.21037 /$ tcr-20-2157

View this article at: http://dx.doi.org/10.21037/tcr-20-2157

\section{Introduction}

Breast cancer (BC) is the most common malignancy and the leading cause of cancer mortality among women worldwide (1). Treatment options for a woman diagnosed with BC include multiple types of treatment (e.g., surgery, chemotherapy and radiation). With the development of medical oncology advances, the therapeutic approaches have changed from a previously incurable condition into a surgical disease. Further improvements in cancer molecular biology allow the opportunities of systemic treatments, including chemotherapy, hormonal therapy and targeted therapy and immunotherapy. Although most BC patients with localized disease are potentially curable with radical surgery, relapse and recurrence in distant organs are incurable and impact long-term prognosis (2). Recurrence and metastasis are key problems affecting the overall survival (OS) of patients with metastatic breast cancer (MBC), which constitutes a major health burden with the highest number of cancer-related deaths among women globally (3). Biological characteristics, such as tumor size, lymph node metastasis, histological grade, age, estrogen receptors (ER), progesterone receptors (PR) and human epidermal growth factor receptor 2 (HER2) status, are considered as traditional prognosis predictor for clinical routine evaluation. However, tumor histological appearance may not be sufficient to establish the underlying complex genetic alterations associated with tumor development and progression. $\mathrm{BC}$ with similar clinical and pathological behaviors may have different biological presentations. Therefore, the heterogeneity of $\mathrm{MBC}$ needs novel biomarkers allowing stratification of patients for treatment despite having similar subtypes; appropriate prognostic factors are urgently needed to predict recurrence risk and prognosis, which will provide effective evidence for the guidance of therapeutic strategy selection for MBC patients.

Emerging evidence suggests that tumor-associated coagulation pathway activation promotes tumor cell proliferation, invasion, and dissemination $(4,5)$. The relationship between malignancy and coagulation system disorders has been explored for more than a century. Fibrinogen is a fundamentally factor in the progression of coagulation pathway activation (6). Increased plasma fibrinogen values were observed in various carcinomas such as gastric cancer, colon cancer, and lung cancer. Recent publications have reported that fibrinogen deficiency diminishes spontaneous hematogenous and lymphatic metastasis according to in vivo and in vitro experiments (7). Previous studies have also demonstrated that plasma fibrinogen is a predictive biomarker for evaluating chemotherapy response in patients with advanced nonsmall-cell lung carcinoma, gastric cancer, and colorectal cancer (8-10). However, the relationship between plasma fibrinogen concentration and the clinical outcomes in patients with MBC is still unknown.

Additionally, tumor development and inflammation status are closely associated (8). In recent years, researchers have found that immune cells (neutrophils, monocytes, and lymphocytes) and platelets are important components of the tumor microenvironment and influence tumor development. The systemic alterations in neutrophil-lymphocyte ratio (NLR) and platelet-lymphocyte ratio (PLR) has come into use recently as an evaluation of inflammatory factors and immune status to better predictions of prognosis $(4,11,12)$. These peripheral blood parameters have been shown to be powerful prognostic factors of poor outcomes in various types of cancers (13-16). A higher preoperative NLR value has been shown to be correlated with poor prognosis in patients with BC (17). Yet, the effect of dynamic change of pre- and post-chemotherapy inflammatory index values on clinical outcomes is still unknown in MBC patients.

Therefore, the present study has been designed to investigate the relationship between the dynamic variation of FIB and the general measures of the immuneinflammation index based on NLR and PLR and to further assess the potential predictive and prognostic role in $\mathrm{MBC}$ patients.

We present the following article in accordance with the STROBE reporting checklist (available at http://dx.doi. org/10.21037/tcr-20-2157).

\section{Methods}

\section{Patient selection}

One hundred and seventy-six patients with $M B C$ who had received first-line therapy at the Affiliated Zhongshan Hospital of Dalian University from June 2011 to March 
2018 were enrolled in this retrospective study. The study methodologies conformed to the provisions of the Declaration of Helsinki (as revised in 2013). This study was reviewed and approved by the Human Ethics Review Committee of the Affiliated Zhongshan Hospital of Dalian University (Protocol: 2020011; 2015032). All enrolled patients signed the written informed consent. Included patients were aged between 24 and 80 years old, with a median age of 56 years. BC staging was based on the seventh edition of the American Joint Committee on Cancer (AJCC-7) Tumor, Node, Metastasis (TNM) staging.

The following inclusion criteria were applied to the selection of patients: (I) invasive BC diagnosed by pathology; (II) MBC baseline treated at the time of enrollment; (III) B-ultrasound, computed tomography (CT), magnetic resonance imaging (MRI), electroconvulsive therapy (ECT), and other examinations were performed prior to treatment to determine the target lesions; (IV) blood sample was collected within one week before treatment; (V) routine follow-up data were available; (VI) patients receiving chemotherapy or targeted therapy should receive at least two cycles of first-line therapy, and patients receiving endocrine and other therapies (zoledronic acid and/or local radiotherapy) should receive at least 2 months of first-line therapy.

The following exclusion criteria were applied: (I) patients with the previous history of thrombosis and long-term oral anticoagulation therapy; (II) patients with severe bleeding or severe infection in the past month; (III) patients with the blood system, bone marrow, or autoimmune diseases; (IV) patients with second malignant tumors; (V) patients with incomplete case information or inaccessible follow-up data.

\section{Patient follow-up}

Patients were followed up via outpatient clinics and telephone contact from the date of blood collection. The follow-up deadline was March 31, 2019. The primary endpoint of follow-up was progression-free survival (PFS) and OS. Response Evaluation Criteria in Solid Tumors (RECIST) were utilized to assess the disease status for each patient with MBC. Patients were grouped as complete response (CR), partial response (PR), stable disease (SD) and disease progression (PD).

\section{Data collection and FIB, NLR, PLR measurements}

One hundred and seventy-six patients were collected peripheral blood samples before first-line therapy; and 56 patients were collected blood samples both baseline and after two cycles of first-line therapy. For fibrinogen measurement, blood samples were collected from fasting patients at 06:00 within 7 days prior to initial treatment; plasma fibrinogen concentrations was measured using an STA Fibrinogen Kit by the Clauss clotting method following the protocol. Normal reference range was defined as between 2 and $4 \mathrm{~g} / \mathrm{L}$. The manufacturer claims an intra assay coefficient of variability (CV) of $2.3-3.7 \%$. In this present study, the mean and median fibrinogen values in total patients were both $4.0 \mathrm{~g} / \mathrm{L}$; therefore, hyperfibrinogenemia was defined as a plasma fibrinogen concentration of $>4 \mathrm{~g} / \mathrm{L}$. For NLR and PLR measurements, blood samples were collected and performed with routine laboratory analysis, including total white blood cell, neutrophil, platelet and lymphocyte counts. The above items were tested in strict accordance with the operating procedures, and the testing environment complies with the relevant operating standards. NLR was defined as the neutrophil/lymphocyte ratio; and PLR was defined as platelet/lymphocyte ratio.

\section{Optimal cut-off values definition}

The optimal cut-off value for FIB was defined as described above. FIB $\geq 4 \mathrm{~g} / \mathrm{L}$ was defined as high FIB group, and FIB $<4 \mathrm{~g} / \mathrm{L}$ was defined as low FIB group. For inflammation-based index, NLR $\geq 2.085$ was defined as high NLR group, and NLR $<2.085$ was defined as low NLR group; PLR $\geq 182$ was defined as high PLR group, and PLR $<182$ was defined as low PLR group. The optimal cutoff points for NLR and PLR were assessed by the area under the receiver operating characteristic (ROC) curve (AUC), which was close to the cutoff values proposed by previous various studies (18).

\section{Statistical analysis}

Data processing and analysis were performed using SPSS20.0 software (SPSS, Chicago, IL, USA). Continuous variables were presented as mean \pm standard deviation, and categorical variables were presented as percentages (\%). Correlations between FIB and NLR or PLR were evaluated using Spearman correlation analysis. Associations between these markers and clinical parameters were evaluated using the Chi-square test analysis. The Kaplan-Meier method, two-tailed log-rank test, and Cox proportional hazard 
Table 1 The relationship between baseline FIB level and clinicopathological features in 176 MBC patients

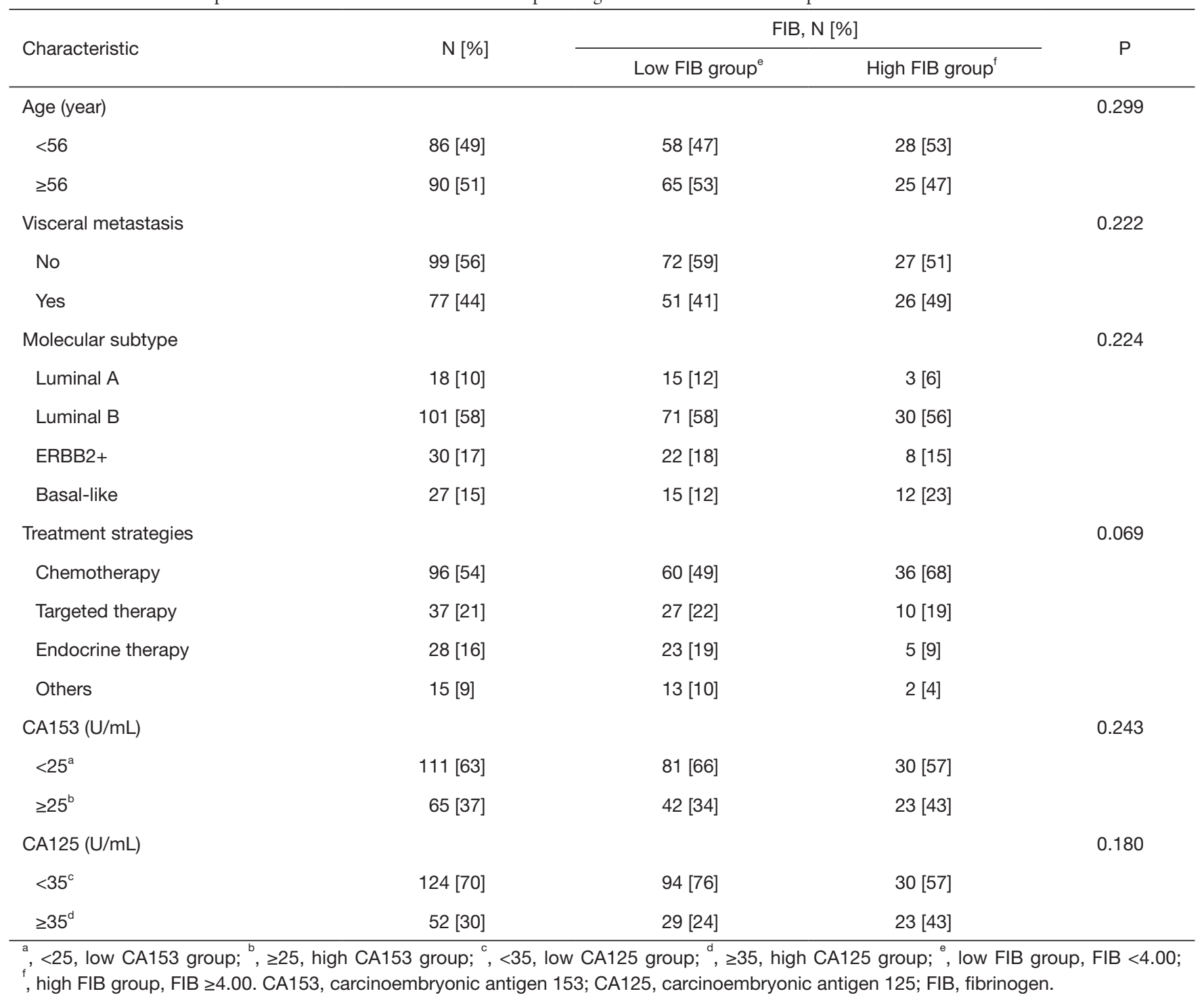

model were utilized for survival analysis. $\mathrm{P}<0.05$ was considered statistically significant.

\section{Results}

\section{The associations between the clinicopathological features and FIB or inflammation-based index in patients with $M B C$}

In total, $176 \mathrm{MBC}$ patients were included in this study. The median age of study participants was 56 years. There were 53 cases in the high-FIB group (30\%) and 123 cases in the low-FIB group (70\%); 77 cases with visceral metastasis
(44\%) and 99 cases without visceral metastasis (56\%); 18 cases with luminal A subtype (10\%), 101 cases with luminal B subtype (58\%), 30 cases with ERBB2 + subtype (17\%), and 27 cases with basal-like subtype (15\%). There were 96 patients undergoing chemotherapy (54\%), 37 patients receiving targeted therapy (21\%), 28 patients receiving endocrine therapy (16\%), and 15 patients being treated with other strategies (9\%).

The associations between FIB or inflammation-based indexes (NLR and PLR) and clinicopathological features in MBC patients are shown in Tables 1 and 2. Baseline fibrinogen levels were not significantly correlated with age $(\mathrm{P}=0.299)$, visceral metastasis $(\mathrm{P}=0.222)$, molecular subtypes 
Table 2 The relationship between baseline inflammation-based index and clinicopathological features in 176 MBC patients

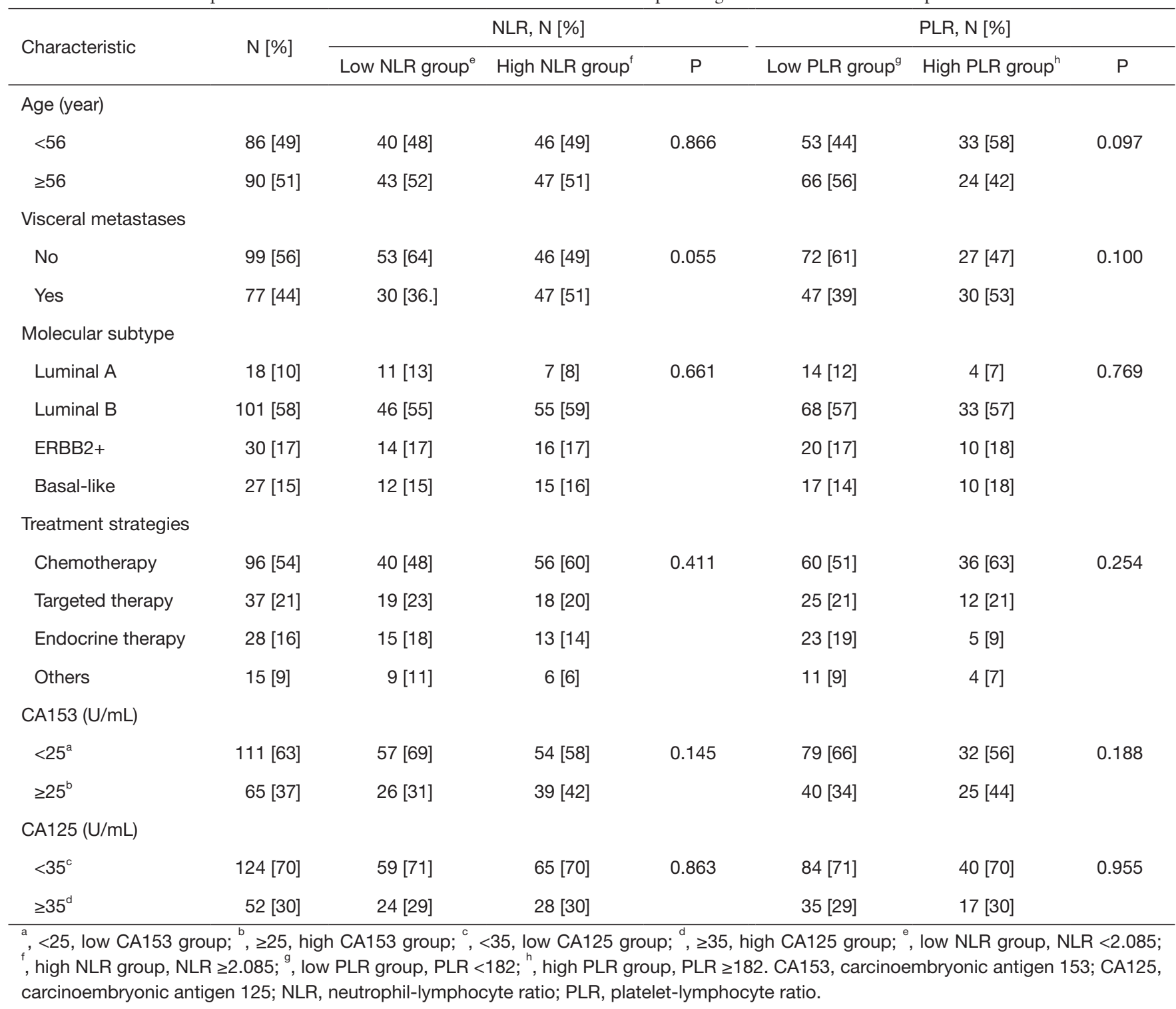

$(\mathrm{P}=0.244)$, therapeutic strategies $(\mathrm{P}=0.069)$, or serum tumor markers $(\mathrm{P}>0.05)$. Also, the NLR and PLR values were not significantly associated with the clinical and laboratory parameters in these patients $(\mathrm{P}>0.05)$.

\section{Survival curve analysis of FIB and inflammation-based index for PFS and OS}

The follow-up deadline was March 31, 2019. The median follow-up time was 25.4 months; 108 of 176 (61\%) MBC patients were observed with disease progression, and 85 of $176(48 \%)$ were followed-up with BC-associated death.
The Kaplan-Meier analysis and log-rank test were then performed to evaluate the survival differences stratified for baseline FIB, NLR, and PLR. As described in Figure $1 A, B$, high baseline FIB status was statistically associated with worse PFS and OS in MBC patients (PFS: $11 \pm 1.8$ vs. $21 \pm 3.5 \mathrm{~m}, \mathrm{P}<0.001$; OS: $22 \pm 1.8$ vs. $44 \pm 5.3 \mathrm{~m}$, $\mathrm{P}<0.001)$. Meanwhile, a higher NLR value (PFS: $15 \pm 1.2$ vs. $22 \pm 5.1 \mathrm{~m}, \mathrm{P}=0.044$; OS: $30 \pm 3.1$ vs. $46 \pm 4.6 \mathrm{~m}, \mathrm{P}=0.003$; Figure $1 C, D)$ and higher PLR value (PFS: $15 \pm 1.4$ vs. $20 \pm 3.3 \mathrm{~m}, \mathrm{P}=0.005$; OS: $28 \pm 3.7$ vs. $43 \pm 2.4 \mathrm{~m}, \mathrm{P}=0.030$; Figure $1 E, F)$ indicated a significant correlation with shorter PFS and survival time. 
A

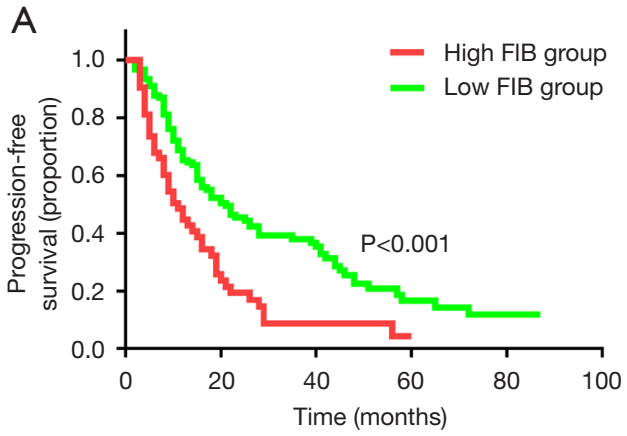

C

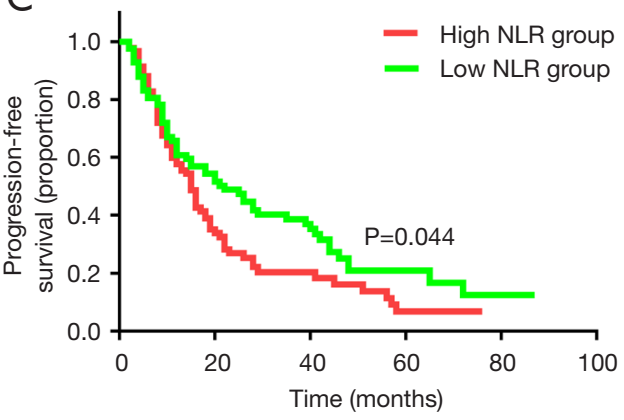

E

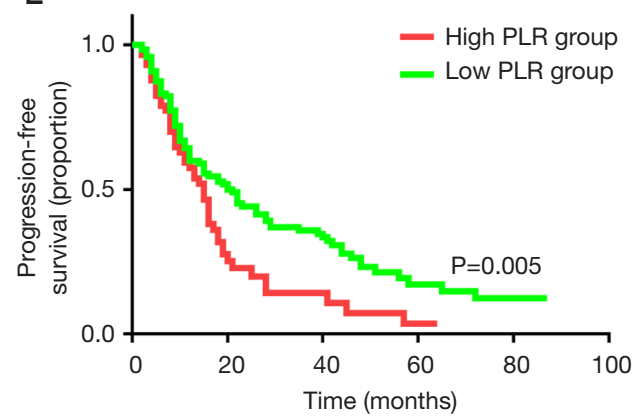

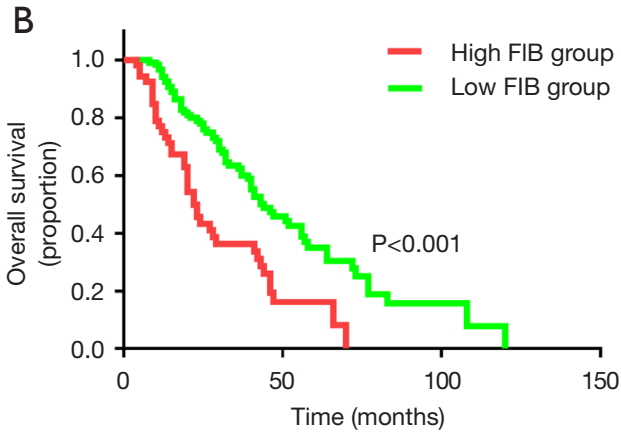

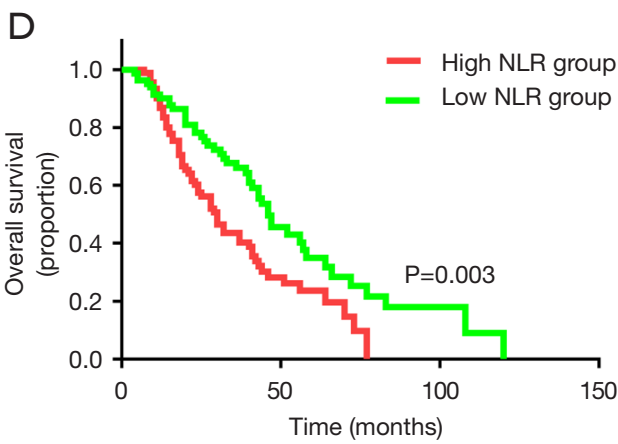

$\mathrm{F}$

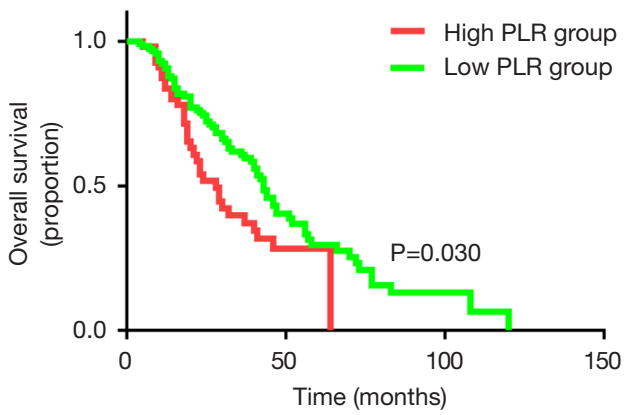

Figure 1 Kaplan-Meier curves for progression-free survival (PFS) and overall survival (OS) according to the baseline FIB level, NLR and PLR values. (A) PFS curves based on FIB level. (B) OS curves based on FIB level. (C) PFS curves based on NLR value. (D) OS curves based on NLR value. (E) PFS curves based on PLR value (F) OS curves based on PLR value. FIB, fibrinogen; NLR, neutrophil lymphocyte ratio; PLR, platelet lymphocyte ratio; high FIB group, FIB $\geq 4$ g/L; low FIB group, FIB <4 g/L; high PLR group, PLR $\geq 182$; low PLR group, PLR <182; high NLR group, NLR $\geq 2.085$; low NLR group, NLR $<2.085$.

\section{Univariate and multivariate analysis for PFS and OS}

Univariate and Cox multivariable proportional hazard models were constructed to evaluate the independent prognostic significance of baseline FIB, NLR, and PLR. Univariate regression analysis showed that visceral metastasis $(\mathrm{P}=0.028)$, basal-like subtype $(\mathrm{P}=0.017)$, endocrine therapy $(\mathrm{P}=0.032)$, other therapy $(\mathrm{P}=0.009)$, FIB
$(\mathrm{P}<0.001)$, and PLR $(\mathrm{P}=0.007)$ were significantly associated with PFS in MBC patients (Table 3). The variables with $\mathrm{P}<0.10$ were included in the multivariate regression analysis using a forward step-wise method.

The results showed that the ERBB2 + subtype ( $\mathrm{P}=0.023)$, basal-like subtype $(\mathrm{P}=0.032)$, targeted therapy $(\mathrm{P}=0.033)$, other therapy $(\mathrm{P}=0.005)$, and FIB $(\mathrm{P}=0.004)$ were 
Table 3 Univariate and multivariate analysis of PFS in 176 patients with MBC

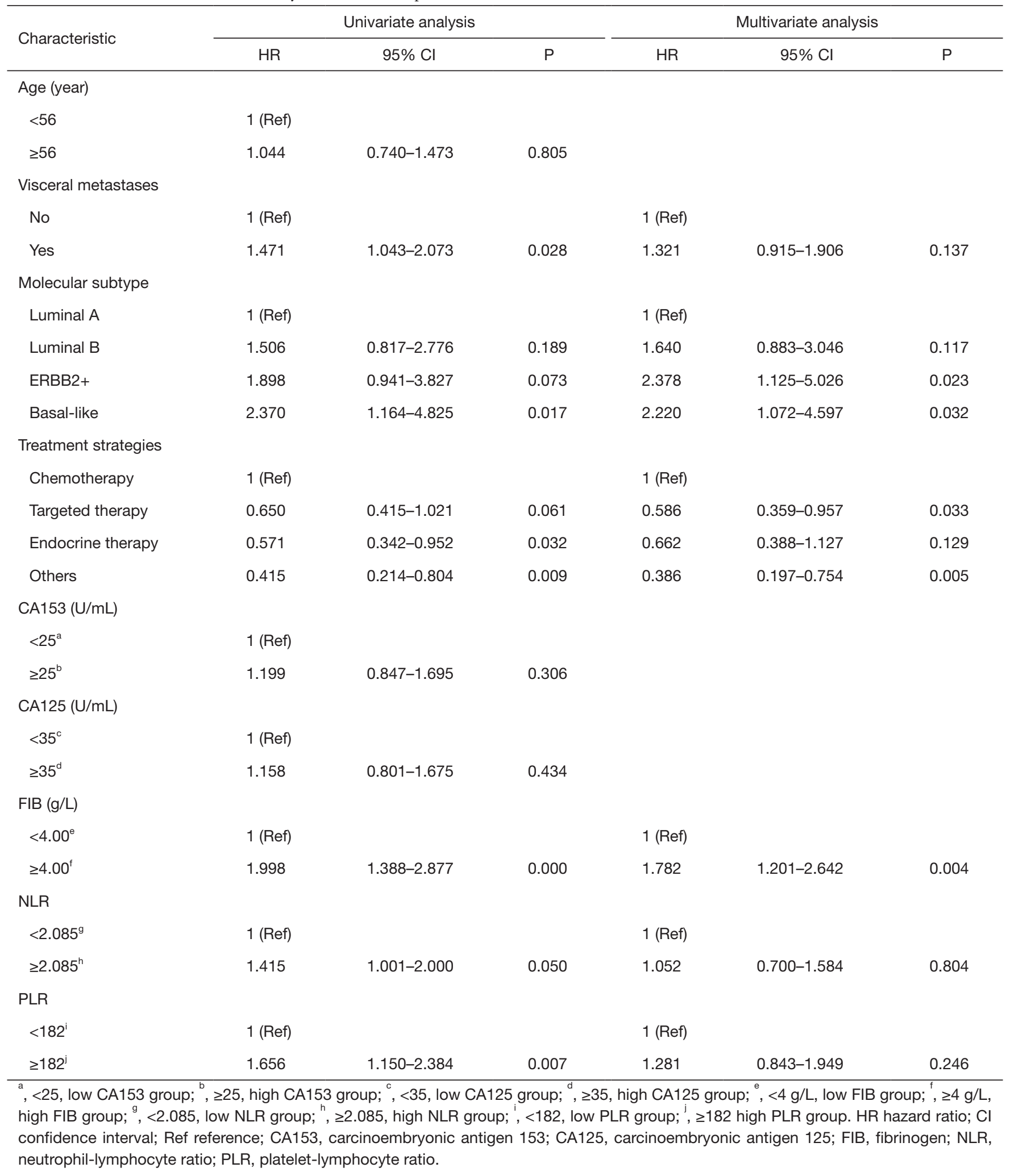


independent factors for PFS in patients with MBC. Next, we assessed whether these variables also had prognostic impacts on OS in MBC. Multivariable analysis of outcomes for the entire cohort showed that ERBB2 + subtypes, basallike subtypes, and baseline hyperfibrinogenemia were independently associated with worse survival [hazard ratio (HR): 3.717, 95\% confidence interval (CI): 1.561-8.851, $\mathrm{P}=0.003$; HR: 3.245 , 95\% CI: $1.368-7.698, \mathrm{P}=0.008$; HR: 2.069, 95\% CI: 1.352-3.167, P=0.001, respectively, Table 4].

\section{Relationship between fibrinogen concentration and the immune-inflammation index}

In order to assess the potential value of FIB for prognosis prediction, we investigated the correlation analysis of baseline FIB level and inflammatory-based scores (NLR and PLR), respectively, for all enrolled MBC patients. As presented in Figure 2A,B, there was a significant positive correlation between FIB and NLR value [correlation coefficient $(r)=0.234, P<0.01]$ and PLR value $(r=0.310$, $\mathrm{P}<0.001)$. The NLR value was also positively correlated with the value of PLR $(\mathrm{r}=0.585, \mathrm{P}<0.001)$ (Figure $2 C$ ). These data indicated that baseline FIB was positively correlated with NLR and PLR values in MBC patients.

\section{Dynamic variation of FIB and correlations with therapeutic response and prognosis}

The changes in FIB before and after treatment were collected from $56 \mathrm{MBC}$ patients to evaluate the relationship between dynamic variation of FIB and therapeutic response and prognosis. Clinical benefit (CB), defined as CR, PR, or $\mathrm{SD}$, was observed in 53 (95\%) of the patients in this cohort. The CR, PR, SD, and PD were two (4\%), 17 (30\%), 34 (61\%), and three (5\%), respectively. As depicted in Table 5 and Figure 3, the mean pre- and post-treatment fibrinogen levels were $3.42 \pm 1.05$ and $3.03 \pm 0.73 \mathrm{~g} / \mathrm{L}$ for the SD group $(\mathrm{P}=0.036)$, and $3.73 \pm 0.63$ and $5.32 \pm 0.52 \mathrm{~g} / \mathrm{L}$ for the PD group $(\mathrm{P}=0.042)$. However, no statistically significant results were observed in other groups $(\mathrm{P}>0.05)$.

Furthermore, these $56 \mathrm{MBC}$ patients were divided into two groups according to the change in pre- and posttreatment fibrinogen levels (cut-off value FIB2-FIB1 $=0 \mathrm{~g} / \mathrm{L}$ ). PFS and OS were not significantly correlated with the increased or decreased fibrinogen groups $(\mathrm{P}=0.685$, $\mathrm{P}=0.249$, respectively, Figure 4).

\section{Dynamic variation of NLR/PLR and correlations with therapeutic response and prognosis}

We subsequently evaluated the relationship between the dynamic change of NLR/PLR indexes, therapeutic response, and prognosis. As presented in Table 6 and Figure 5, there were no significant differences between the dynamic variations of NLR/PLR indexes and therapeutic response in $\mathrm{MBC}$ patients $(\mathrm{P}>0.05)$.

Fifty-six MBC patients were divided into four groups according to the change in pre- and post-treatment NLR and PLR values (cut-off value NLR2-NLR1 $=0$; PLR2PLR1 =0). Both PFS and OS were not significantly associated with the change of NLR or PLR value $(P>0.05$, Figure 6). These data indicated that the dynamic change of NLR/PLR values was not correlated with therapeutic response and prognosis in $\mathrm{MBC}$ patients.

\section{Discussion}

$\mathrm{MBC}$ is a heterogeneous disease with various biological behaviors, natural history, therapeutic response and prognosis $(19,20)$. The clinical parameters that have been reported to predict outcomes of patients with MBC include the TNM stage, Eastern Cooperative Oncology Group (ECOG) score, histopathological grade, and molecular subtypes (21). However, the progression of tumor cells is not only determined by pathological characteristics but is also influenced by baseline biochemical and hematological parameters $(5,22)$. Yet, it is still difficult to predict and evaluate the metastatic risk for patients with occult metastasis based on imaging examination. Hence, a convenient and dynamic method of predicting relapse risk and monitoring a therapeutic response is urgently needed. In this present study, we reported, for the first time, that baseline FIB and the inflammation-based index were independent prognostic factors in patients with MBC.

Fibrinogen is a critical indicator in the coagulation pathway, which functions as the final step of the coagulation cascade (23). In addition to its well-known role in blood coagulation, it has been increasingly recognized that a close link exists between hyperfibrinogenemia and various malignancies, including breast, gastric, lung, and colorectal cancers (8-10). Hyperfibrinogenemia status plays an essential role in the progression of tumors, such as providing a microenvironment for tumor cell proliferation and protecting tumors cells from a systemic immune 
Table 4 Univariate and multivariate analysis of OS in 176 patients with MBC

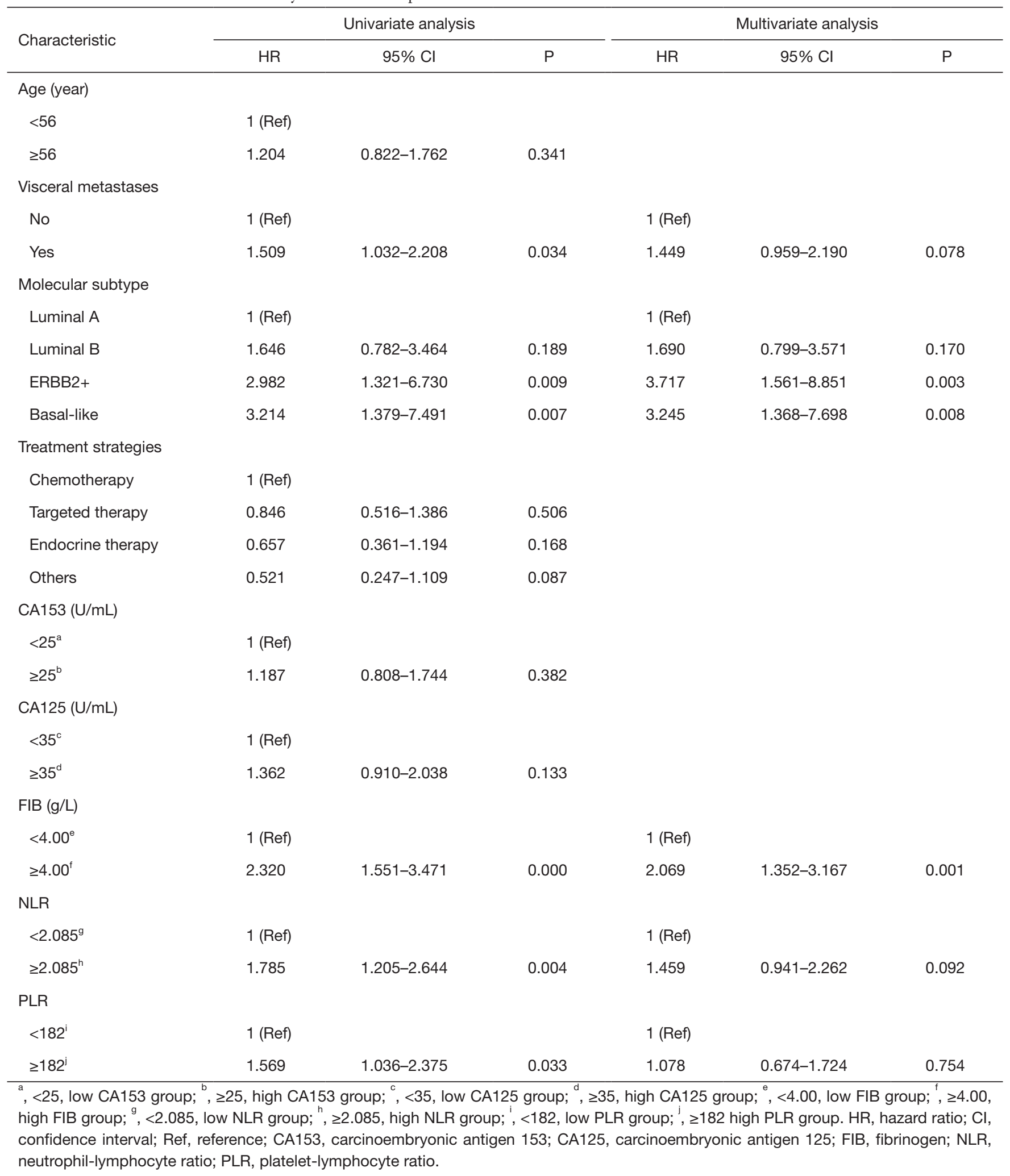



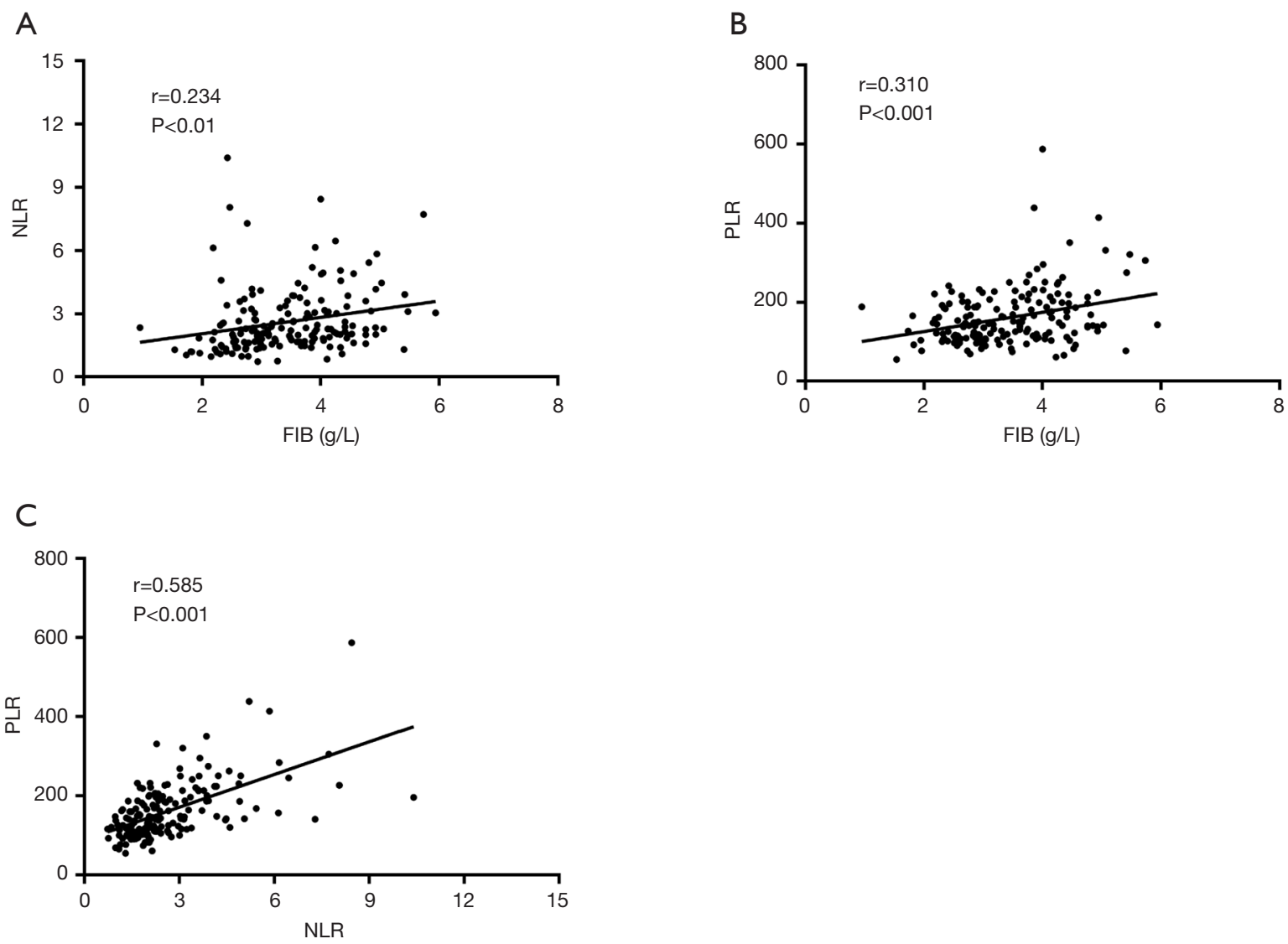

Figure 2 Spearman correlation analysis between FIB and inflammation-based indexes. The scatter plots represent association of FIB levels and NLR (A), relationship between FIB level and PLR (B) and correlation between NLR and PLR (C) in MBC patients. FIB, fibrinogen; NLR, neutrophil lymphocyte ratio; PLR, platelet lymphocyte ratio.

Table 5 Variation of FIB level pre- and post-treatment and correlation with therapeutic response

\begin{tabular}{|c|c|c|c|c|}
\hline Variables & $\mathrm{N}[\%]$ & Pre-treatment FIB (g/L) & Post-treatment FIB (g/L) & $\mathrm{P}$ \\
\hline PR & 17 [30] & $3.04 \pm 0.96$ & $3.00 \pm 0.77$ & 0.855 \\
\hline SD & $34[61]$ & $3.42 \pm 1.05$ & $3.03 \pm 0.73$ & 0.036 \\
\hline PD & 3 [5] & $3.73 \pm 0.63$ & $5.32 \pm 0.52$ & 0.042 \\
\hline Total & 56 & $3.32 \pm 0.99$ & $3.12 \pm 0.89$ & 0.156 \\
\hline
\end{tabular}

CR complete relief; PR, partial relief; SD, stable disease; PD, progressive disease; DCR, disease control rate; FIB, fibrinogen.

response, which may potentially impact the long-term prognosis for cancer patients $(24,25)$. In the present study, baseline hyperfibrinogenemia tended to be an independent marker of poor prognosis in MBC patients, which is in line with the previously published research for $\mathrm{BC}$ and other malignancies $(9,26)$. However, no data has been reported on the dynamic change of fibrinogen and the prognostic value in patients with MBC. To the best of our knowledge, this is the first report indicating that the dynamic variation of FIB predicts the response to first-line therapy in patients 
with MBC.

Most importantly, the prognostic inflammatory index (NLR and PLR scores) was examined in combination with the fibrinogen value simultaneously in this study. It is well established that chronic environment-induced inflammation is the main cause leading to the occurrence of cancer $(5,27)$. Crosstalk between circulating inflammatory immune cells and tumor cells forms a cancer microenvironment that promotes tumor progression (28). It has been recognized that lymphocytes play a key role in immune-editing and immune surveillance, which inhibits the invasion and proliferation of tumor cells. Moreover, platelets are associated with poor cancer prognosis due to the induction of platelet-derived growth factor and vascular endothelial growth factor, both of which promote tumor cell proliferation and invasion $(4,29)$. Therefore, indicators of chronic inflammation, such as NLR and PLR, may be

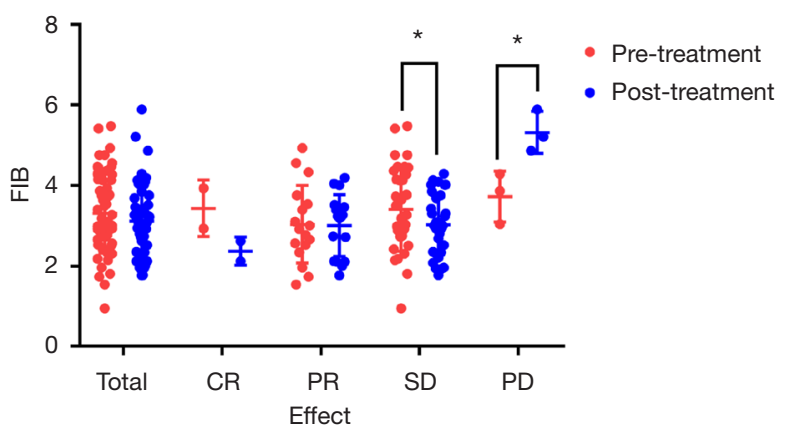

Figure 3 Comparison between pre- and post-treatment FIB levels in $\mathrm{MBC}$ patients. CR, complete response; $\mathrm{PR}$, partial response; $\mathrm{SD}$, stable disease; $\mathrm{PD}$, disease progression; DCR, disease control rate, $\mathrm{CR}+\mathrm{PR}+\mathrm{SD}$. *, $\mathrm{P}<0.05$. candidate biomarkers for predicting the clinical outcome of cancer patients.

Previous studies have shown that both NLR and PLR are valuable as predictors of $\mathrm{BC}$ prognosis and therapeutic response (17). However, the role of inflammation indexes in MBC is controversial, and dynamic changes of NLR and PLR scores and prognostic value in patients with MBC are still lacking. Thus, we combined the serum fibrinogen, NLR and PLR together to assess the association with clinicopathological characteristics, therapeutic response and prognosis. To the best of our knowledge, this is the first clinical study to evaluate the potential value in MBC patients. In our study, Spearman rank correlation analysis revealed a significant correlation between fibrinogen concentration and NLR and PLR counts. In addition, we assessed the relationship between the variation of inflammatory indexes before and after treatment and therapeutic response, but no significantly different conclusions were surmised from this data. Therefore, as non-invasive, convenient and easily acquired measurements in clinical practice, detection of both baseline FIB and inflammation indexes were novel clinical biomarkers and had powerful prognostic values for MBC. It is important to note that this study exhibited the inevitable limitations of retrospective studies conducted at a single center with a smaller sample size. Therefore, our findings require validation with large-scale, multicenter, controlled clinical studies.

\section{Conclusions}

In conclusion, baseline FIB is an independent predictor of prognosis in patients with MBC. Dynamic change of
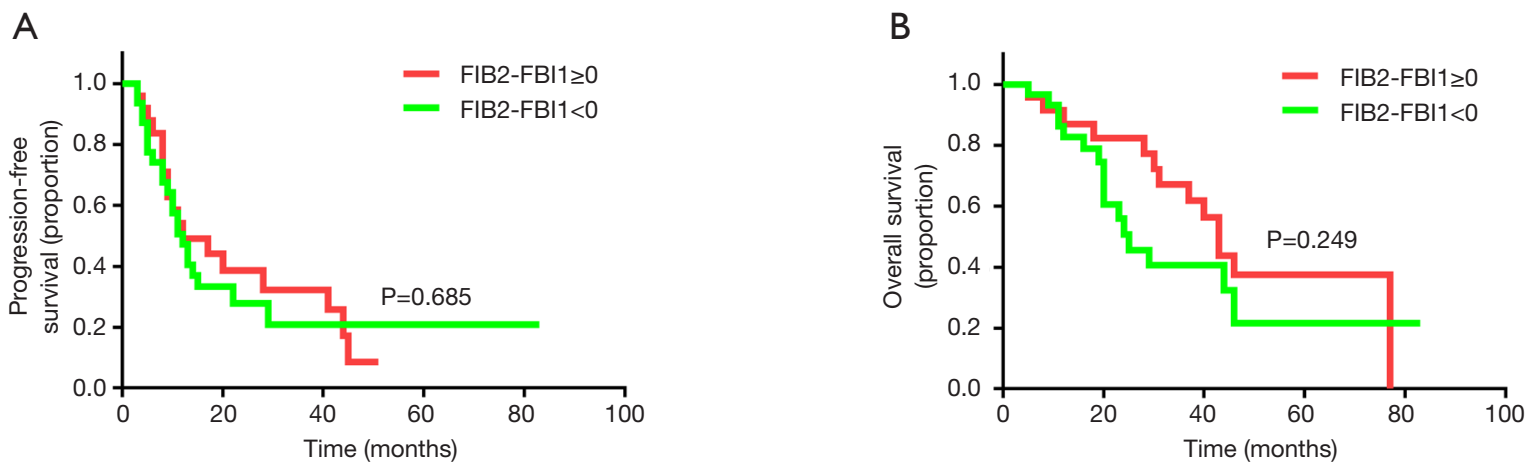

Figure 4 Kaplan-Meier curves for progression-free survival (PFS) (A) and overall survival (OS) (B) according to the pre- and post-treatment FIB levels. FIB2, post-treatment FIB level; FIB1, pre-treatment FIB level. 
Table 6 Variation of inflammation-based index pre- and post-treatment and correlation with therapeutic response

\begin{tabular}{|c|c|c|c|c|c|c|c|}
\hline Variables & $\mathrm{N}$ & \multicolumn{3}{|c|}{ NLR } & \multicolumn{3}{|c|}{ PLR } \\
\hline CR & 2 & $1.50 \pm 1.08$ & $1.10 \pm 0.33$ & 0.594 & $113.04 \pm 4.29$ & $78.48 \pm 17.43$ & 0.266 \\
\hline PR & 17 & $2.22 \pm 1.11$ & $1.72 \pm 0.97$ & 0.108 & $135.21 \pm 49.50$ & $122.04 \pm 40.69$ & 0.243 \\
\hline SD & 34 & $2.45 \pm 1.29$ & $2.32 \pm 1.27$ & 0.618 & $176.19 \pm 70.60$ & $171.64 \pm 62.11$ & 0.713 \\
\hline DCR & 53 & $2.34 \pm 1.23$ & $2.08 \pm 1.20$ & 0.182 & $160.66 \pm 66.13$ & $152.88 \pm 60.84$ & 0.374 \\
\hline Total & 56 & $2.39 \pm 1.25$ & $2.37 \pm 1.95$ & 0.931 & $164.43 \pm 74.74$ & $167.78 \pm 125.99$ & 0.794 \\
\hline
\end{tabular}

CR, complete relief; PR, partial relief; SD, stable disease; PD, progressive disease; DCR, disease control rate; NLR, neutrophil-lymphocyte ratio; PLR, platelet-lymphocyte ratio.
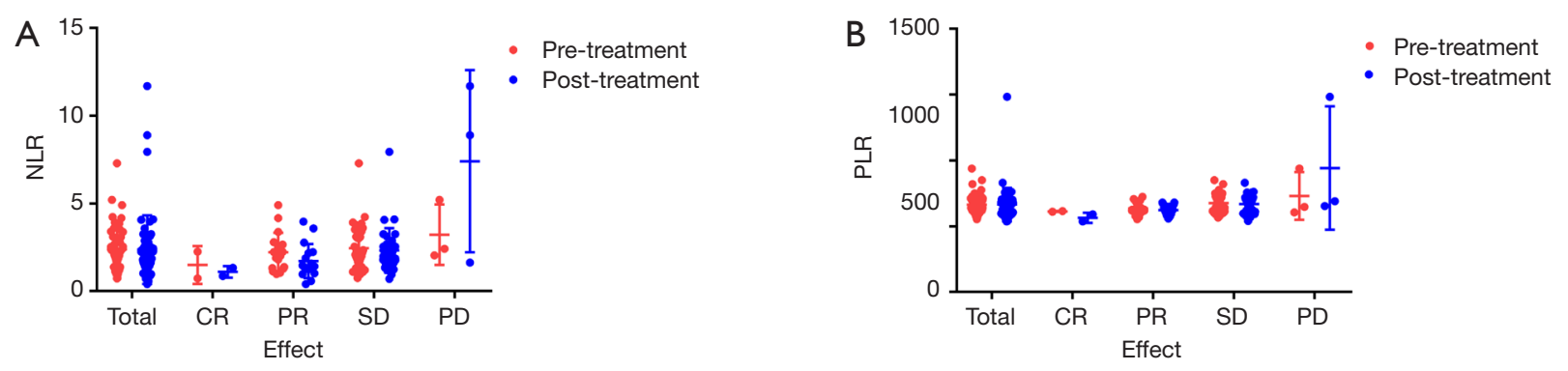

Figure 5 Comparison between pre- and post-treatment NLR value (A) and PLR value (B) in MBC patients. CR, complete response; PR, partial response; $\mathrm{SD}$, stable disease; $\mathrm{PD}$, disease progression.
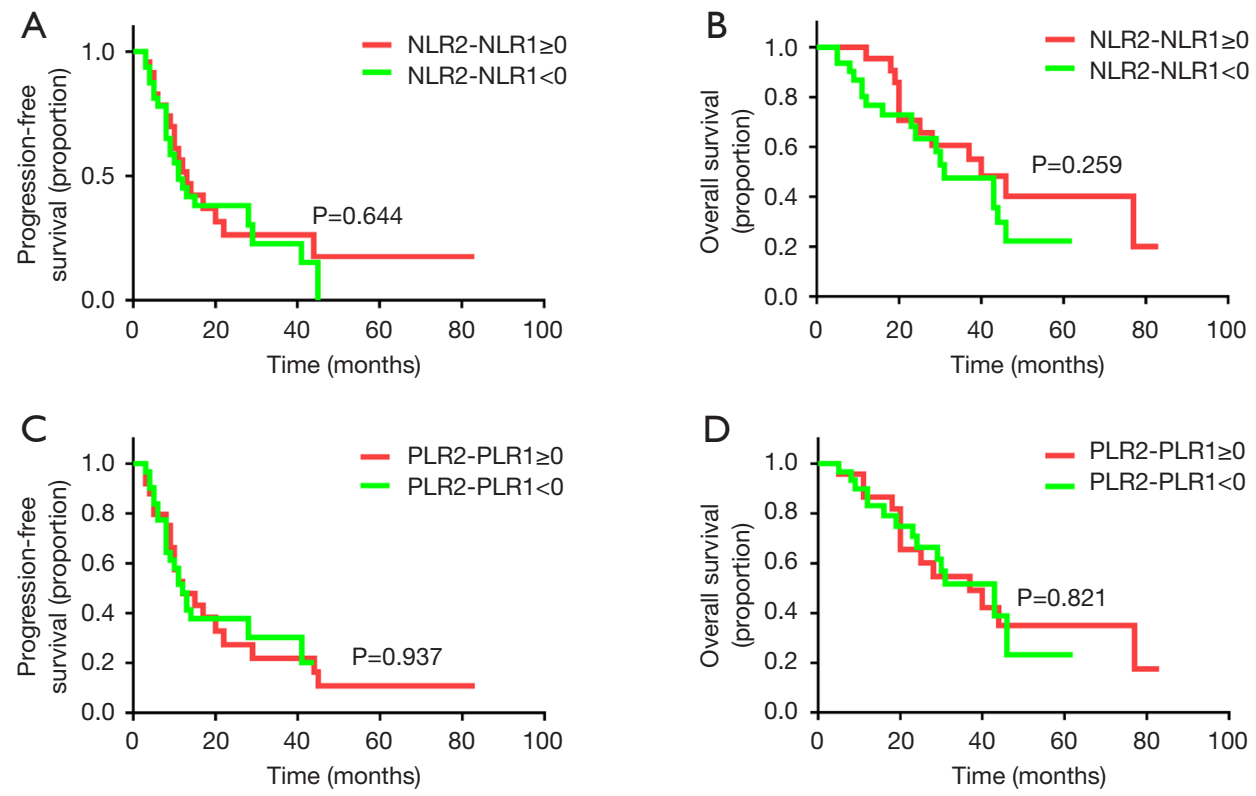

Figure 6 Kaplan-Meier curves for progression-free survival (PFS) (A,C) and overall survival (OS) (B,D) according to the pre- and posttreatment NLR value (A,B) and PLR value (C,D). NLR2, post-treatment NLR value; NLR1, pre-treatment NLR value; PLR2, posttreatment PLR value; PLR1, pre-treatment PLR value. 
fibrinogen levels before and after therapy is associated with the therapeutic response. Plasma fibrinogen would be a useful biomarker of prognosis in MBC patients because it is inexpensive and readily accessible. However, the value of monitoring FIBs in patients with MBC requires further evaluation.

\section{Acknowledgments}

Funding: The study was supported by grants from Natural Science Foundation of Liaoning Province (Grant 2019-MS010); Dalian Medical and Science Research Fund Project (Grant 1911096); Chinese Society of Clinical Oncology Research Funding (Grant Y-HR2018-117).

\section{Footnote}

Reporting Checklist: The authors have completed the STROBE reporting checklist. Available at http://dx.doi. org/10.21037/tcr-20-2157

Data Sharing Statement: Available at http://dx.doi. org/10.21037/tcr-20-2157

Conflicts of Interest: All authors have completed the ICMJE uniform disclosure form (available at http://dx.doi. org/10.21037/tcr-20-2157). The authors have no conflicts of interest to declare.

Ethical Statement: The authors are accountable for all aspects of the work in ensuring that questions related to the accuracy or integrity of any part of the work are appropriately investigated and resolved. This study was approved by the Ethics Committee of The Affiliated Zhongshan Hospital of Dalian University (Protocol: 2020011; 2015032). Signed written informed consent was obtained from the patient who was collected the blood sample. The study methodologies conformed to the provisions of the Declaration of Helsinki (as revised in 2013), available at: https://www.wma.net/wp-content/ uploads/2016/11/DoH-Oct2013-JAMA.pdf.

Open Access Statement: This is an Open Access article distributed in accordance with the Creative Commons Attribution-NonCommercial-NoDerivs 4.0 International License (CC BY-NC-ND 4.0), which permits the noncommercial replication and distribution of the article with the strict proviso that no changes or edits are made and the original work is properly cited (including links to both the formal publication through the relevant DOI and the license). See: https://creativecommons.org/licenses/by-nc-nd/4.0/.

\section{References}

1. Siegel RL, Miller KD, Jemal A. Cancer statistics, 2019. CA Cancer J Clin 2019;69:7-34.

2. Woo JW, Chung YR, Ahn S, et al. Changes in Biomarker Status in Metastatic Breast Cancer and Their Prognostic Value. J Breast Cancer 2019;22:439-52.

3. Peart O. Metastatic Breast Cancer. Radiol Technol 2017;88:519M-539M.

4. Mantovani A, Allavena P, Sica A, et al. Cancer-related inflammation. Nature 2008;454:436-44.

5. Grivennikov SI, Greten FR, Karin M. Immunity, inflammation, and cancer. Cell 2010;140:883-99.

6. Lima LG, Monteiro RQ. Activation of blood coagulation in cancer: implications for tumour progression. Biosci Rep 2013;33:e00064.

7. Palumbo JS, Potter JM, Kaplan LS, et al. Spontaneous hematogenous and lymphatic metastasis, but not primary tumor growth or angiogenesis, is diminished in fibrinogendeficient mice. Cancer Res 2002;62:6966-72.

8. Hou C, Jiang F, Ma H, et al. Prognostic role of preoperative platelet, fibrinogen, and D-dimer levels in patients with non-small cell lung cancer: A multicenter prospective study. Thorac Cancer 2019;10:304-11.

9. Palaj J, Kečkéš Š, Marek V, et al. Fibrinogen Levels Are Associated with Lymph Node Involvement and Overall Survival in Gastric Cancer Patients. Anticancer Res 2018;38:1097-104.

10. Li M, Wu Y, Zhang J, et al. Prognostic value of pretreatment plasma fibrinogen in patients with colorectal cancer: A systematic review and meta-analysis. Medicine (Baltimore) 2019;98:e16974.

11. Sun Y, Zhang L. The clinical use of pretreatment NLR, PLR, and LMR in patients with esophageal squamous cell carcinoma: evidence from a meta-analysis. Cancer Manag Res 2018;10:6167.

12. Wang H, Zhao J, Zhang M, et al. The combination of plasma fibrinogen and neutrophil lymphocyte ratio (F-NLR) is a predictive factor in patients with resectable non small cell lung cancer. J Cell Physiol 2018;233:4216-24.

13. Diem S, Schmid S, Krapf M, et al. Neutrophil-toLymphocyte ratio (NLR) and Platelet-to-Lymphocyte ratio (PLR) as prognostic markers in patients with non- 
small cell lung cancer (NSCLC) treated with nivolumab. Lung Cancer 2017;111:176-81.

14. Hirahara T, Arigami T, Yanagita S, et al. Combined neutrophil-lymphocyte ratio and platelet-lymphocyte ratio predicts chemotherapy response and prognosis in patients with advanced gastric cancer. BMC Cancer 2019;19:672.

15. Bilen MA, Martini DJ, Liu Y, et al. The prognostic and predictive impact of inflammatory biomarkers in patients who have advanced-stage cancer treated with immunotherapy. Cancer 2019;125:127-34.

16. Ying HQ, Deng QW, He BS, et al. The prognostic value of preoperative NLR, d-NLR, PLR and LMR for predicting clinical outcome in surgical colorectal cancer patients. Med Oncol 2014;31:305.

17. Duan J, Pan L, Yang M. Preoperative elevated neutrophil-to-lymphocyte ratio (NLR) and derived NLR are associated with poor prognosis in patients with breast cancer: A meta-analysis. Medicine (Baltimore) 2018;97:e13340.

18. Fang T, Wang Y, Yin X, et al. Diagnostic Sensitivity of NLR and PLR in Early Diagnosis of Gastric Cancer. J Immunol Res 2020;2020:9146042.

19. Yersal O, Barutca S. Biological subtypes of breast cancer: Prognostic and therapeutic implications. World J Clin Oncol 2014;5:412.

20. Kartolo A, Holstead R, Khalid S, et al. Serum neutrophilto-lymphocyte ratio and platelet-to-lymphocyte ratio in prognosticating immunotherapy efficacy. Immunotherapy 2020;12:785-98.

Cite this article as: Liu Q, Fang S, Liang S, Lv J, Wang G, Tang R, Ji X, Zhao T, Li J, Xu L, Ma L, Wang R, Li H. The prognostic role of a combined fibrinogen and inflammationbased index in patients with metastatic breast cancer. Transl Cancer Res 2020;9(11):7065-7078. doi: 10.21037/tcr-20-2157
21. Soerjomataram I, Louwman MW, Ribot JG, et al. An overview of prognostic factors for long-term survivors of breast cancer. Breast Cancer Res Treat 2008;107:309-30.

22. Palumbo JS, Talmage KE, Massari JV, et al. Tumor cellassociated tissue factor and circulating hemostatic factors cooperate to increase metastatic potential through natural killer cell-dependent and-independent mechanisms. Blood 2007;110:133-41.

23. Kamath S, Lip G. Fibrinogen: biochemistry, epidemiology and determinants. QJM 2003;96:711-29.

24. Palumbo JS, Degen JL. Mechanisms linking tumor cellassociated procoagulant function to tumor metastasis. Thromb Res 2007;120:S22-8.

25. Palumbo JS, Barney KA, Blevins EA, et al. Factor XIII transglutaminase supports hematogenous tumor cell metastasis through a mechanism dependent on natural killer cell function. J Thromb Haemost 2008;6:812-9.

26. Dirix LY, Salgado R, Weytjens R, et al. Plasma fibrin D-dimer levels correlate with tumour volume, progression rate and survival in patients with metastatic breast cancer. Br J Cancer 2002;86:389-95.

27. Baniyash M, Sade-Feldman M, Kanterman J. Chronic inflammation and cancer: suppressing the suppressors. Cancer Immunol Immunother 2014;63:11-20.

28. Qu X, Tang Y, Hua S. Immunological approaches towards cancer and inflammation: a cross talk. Front Immunol 2018;9:563.

29. Balkwill F, Mantovani A. Inflammation and cancer: back to Virchow? Lancet 2001;357:539-45. 